\title{
ILCEA
}

Revue de l'Institut des langues et cultures

d'Europe, Amérique, Afrique, Asie et Australie

17 | 2013

1861-2011 : réflexions sur l'abolition du servage en

Russie

\section{L'émancipation des serfs en Russie : les projets en français (1858-1861)}

Emancipation of the Serfs in Russia: projects in French (1858-1861)

ОТМЕНА КРЕПОСТНОГО ПРАВА А РОССИИ: ПРОЕКТЫ ПО-ФРАНЦУЗСКИ (1858-1861)

\section{Michel Niqueux}

\section{OpenEdition}

\section{Journals}

Édition électronique

URL : http://journals.openedition.org/ilcea/1751

DOI : 10.4000/ilcea.1751

ISSN : 2101-0609

\section{Éditeur}

UGA Éditions/Université Grenoble Alpes

Édition imprimée

ISBN : 978-2-84310-243-1

ISSN : 1639-6073

\section{Référence électronique}

Michel Niqueux, «L'émancipation des serfs en Russie : les projets en français (1858-1861) », ILCEA [En ligne], 17 | 2013, mis en ligne le 31 janvier 2013, consulté le 20 avril 2019. URL : http:// journals.openedition.org/ilcea/1751 ; DOI : 10.4000/ilcea.1751

Ce document a été généré automatiquement le 20 avril 2019.

(c) ILCEA 


\title{
L'émancipation des serfs en Russie : les projets en français (1858-1861)
}

\author{
Emancipation of the Serfs in Russia: projects in French (1858-1861) \\ ОТМЕНА КРЕПОСТНОГО ПРАВА А РОССИИ: ПРОЕКТЫ ПО-ФРАНЦУЗСКИ (1858-1861)
}

\section{Michel Niqueux}

1 La préparation de l'« émancipation des serfs ${ }^{1}$ » donna lieu, après les rescrits de décembre 1857 sur l'« amélioration de la situation des paysans ", à une discussion publique d'une ampleur sans précédent, à un premier exemple de glasnost', bien encadrée cependant par la censure.

\section{Les limites de la glasnost'}

2 En mars 1857, Schédo-Ferroti (pseudonyme du baron balte Fëdor Ivanovitch Firks, 1812-1872, auteur d'une dizaine d'Études sur l'avenir de la Russie, 1857-1868), regrettait encore l'absence de libre discussion et une censure qui interdisait les ouvrages sérieux sur les questions sociales, tout en permettant « le débit des comédies, romans et contes qui font la critique des mœurs de l'époque ", "de tous ces ouvrages de l'école moderne, dont le chef de file est Mr. Gogol, et qui prend à tâche de dévoiler les plaies les plus hideuses de la société » $(1$, p. 28, 27) 2 . La situation change après la publication des rescrits, fin 1857. Dès le début de l'année 1858, journaux et revues s'emparent du sujet. Des revues spécialisées sont créées (Sel'skoe blagoustrojstvo de A. I. Kochelev, Žurnal zemlevladel'cev³). Toutefois, des limites précises sont imposées par la censure :

- Les articles qui analyseront, discuteront et critiqueront les dispositions du gouvernement sur la question [« la libération de l'état de servage »] seront interdits de publication ;

- Seront aussi interdits les articles, principalement littéraires, où sous forme de récit ou autre renferment des événements ou des opinions pouvant exciter les paysans contre les propriétaires ;

- Les ouvrages ou articles purement savants, théoriques, historiques et statistiques [ne seront autorisés qu'à condition] a) de ne pas contenir de commentaires et d'interprétations sur les 
principes fondamentaux des rescrits $[. .$.$] ; b) de respecter les règles générales de la censure ;$ c) de prêter une attention particulière à l'esprit et aux bonnes intentions du texte ; d) les articles écrits dans l'esprit du gouvernement peuvent être publiés dans toutes les revues ${ }^{4}$.

En février 1858, il sera prescrit au comité de censure de Moscou d'interdire «tout ce qui transporte la question paysanne sur le sol politique, en maintenant les écrivains dans les limites indiquées par le gouvernement ${ }^{5} »$. De plus, les articles devaient être préalablement soumis aux ministères et administrations dont relevaient les questions qu'ils traitaient, le visa du ministère ne préjugeant pas de celui de la censure ${ }^{6}$. En avril 1858, le Russkij vestnik préféra ainsi interrompre sa rubrique sur «La question paysanne ${ }^{7}$ », et le Sel'skoe blagoustrojstvo de A. I. Kochelev dut cesser de paraître après le numéro de février 1859 en raison de ces fourches caudines8

4 La glasnost' a donc ses limites, comme le ministre de l'Instruction publique le rappelait dans une circulaire du 3 avril 1859 : la glasnost peut être utile quand elle révèle des désordres ou des abus ${ }^{9}$, car

elle donne au Gouvernement la possibilité d'être informé indépendamment des sources officielles [...] et de prendre des mesures adéquates. Mais la glasnot peut aussi être nuisible, quand elle touche à d'importants sujets d'administration, qui n'ont pas encore été définitivement examinés par le Gouvernement, ou qu'il ne considère pas comme dignes d'attention ${ }^{10}$.

5 Les centaines de projets qui remontèrent des comités de rédaction provinciaux au comité principal n'étaient pas destinés à être diffusés et échappaient donc à ces règles. Le comité principal prit en considération « tout ce qui avait été publié en Russie et à l'étranger, en livres, brochures ou en articles de journaux » et rassembla les projets en vingt-six gros volumes.En janvier 1860, Sazonov, ancien membre du Membre du cercle étudiant de Herzen et Ogarev à Moscou, écrivait avec emphase dans son journal La Gazette du Nord, ( ${ }^{\circ}$ 1 du 7 janvier 1860), favorable à Alexandre II, mais sans servilisme :

C'est là un beau et grand spectacle, nous le disons avec un sentiment de fierté nationale, que de voir dans un grand pays, pour la première fois s'essayant à l'usage de la libre discussion, tous les hommes les plus intelligents, se vouer avec un zèle infatigable et, pour la plupart avec une remarquable bonne foi, à l'étude de cette énorme question qui porte dans son sein l'avenir de la Russie et celui du monde.

Cependant, dans les conditions de la censure rappelées ci-dessus, on comprend que nombre d'auteurs aient préféré choisir de se faire publier à l'étranger, même si tous leurs écrits étaient loin d'être des brûlots comme $L a$ vérité de la Russie du prince Piotr Dolgoroukov, auquel Herzen écrivait en mars 1860 : «Vous faites très bien de publier en français, ils [le gouvernement russe] craignent cela comme le feu ${ }^{11}$. Il y a aussi le désir d'informer l'opinion publique européenne, d'élargir le débat, de faire ainsi pression sur le gouvernement russe. Nous avons ainsi trouvé à la BNF et à la Bibliothèque slave de l'ENS de Lyon près de quarante brochures ou ouvrages en français traitant de l'abolition du servage. La liste jointe à la fin de cet article n'est toutefois pas exhaustive : il manque des brochures, la presse et les périodiques, à part la Revue des deux mondes et La Gazette du Nord , n'ont pas été déoouillés ${ }^{12}$. Ces projets en français, publiés à l'étranger (en France et dans d'autres pays) n'ont pas fait l'objet d'un inventaire et n'ont pas été spécialement étudiés, seul Nicolas Tourguenev, l'auteur le plus connu, étant souvent cité.

7 Il s'agit de projets en bonne et due forme, d'exposés des réformes en cours, plus ou moins critiques, généralement précédés d'un historique du servage, ou encore de réflexions sur la question de l'émancipation. Les auteurs sont soit des émigrés russes (Nicolas Tourguenev, Augustin Galitzine, Piotr Dolgoroukov), soit des Russes publiant à l'étranger 
(Dmitri Stolypine, Schedo-Ferroti), des Polonais, ou des observateurs étrangers (le prince Henri de Valori [1831-1898] estimant que "seul un publiciste français peut examiner froidement la question russe» $[26$, p. 6]). Beaucoup de brochures sont anonymes («un député », « un publiciste», " un panslaviste», «un Russe »...) ou publiées sous pseudonyme, pour des raisons qui tiennent plus souvent, probablement, aux habitudes de l'époque qu'à de la pusillanimité. Mais le "paysan obligé » qui se référe à Herzen, "l'apôtre russe de la vérité et de la justice ", pour rejeter la création d'un " prolétariat " et affirmer « La terre nous appartient ! » est-il vraiment un paysan ? $(29$, p. 6$)$.

En janvier 1859, Nicolas de Gerebtzoff (1807-1868), conseiller d'État actuel, ancien gouverneur de Vilno, qui avait publié l'année précédente (en France) une somme de près 1200 pages, en deux volumes, sur l'Histoire de la civilisation en Russie, d'inspiration patriotique et slavophile mais bien documentée, regrettait ce recours à l'anonymat :

Depuis quelque temps, les hautes questions politiques à l'ordre du jour sont traitées dans des brochures : la publicité des journaux paraitt insuffisante aux propagateurs de systèmes plus ou moins excentriques, et la Russie est devenue l'objet principal de leurs commentaires. Aussi les grands problèmes sociaux, qui se débattent actuellement dans ce pays, donnent-ils matière à une foule de brochures, presque toutes anonymes, quoique l'anonyme soit toujours un signe de faiblesse dans les convictions ou d'idées qu'on n'ose pas avouer. (11, p. 5)

Le plus connu, après Nicolas Tourguenev, des émigrés russes ayant écrit en français sur le servage est le prince Piotr Dolgoroukov (1816-1868). Généalogiste, publiciste, historien personnage sulfureux, aux ambitions contrariées, craint ou honni pour ses révélations sur les secrets et les turpitudes de l'aristocratie, il est l'un des principaux représentants du libéralisme russe, qui n'en compte pas tellement ${ }^{13}$.

En 1841, il part pour Paris, où il publie en 1842, en français, sous le nom de comte d'Almagro une Notice sur les principales familles de la Russie, recueil de données généalogiques et de ce qu'on appelerait maintenant du kompromat sur les dignitaires de l'époque de Nicolas $\mathrm{I}^{\text {er }}$ et la dynastie des Romanov. Revenu en 1843 en Russie, il fut assigné un an à résidence à Viatka ${ }^{14}$. En 1859, il quitta secrètement la Russie et publia en 1860, toujours en français, La vérité sur la Russie, tableau de la Russie qui reste malheureusement actuel (il fut interdit d'introduction en France à la demande du gouvernement russe). Dolgoroukov passe en revue toutes les institutions et les corps sociaux de la Russie, en proposant des réformes. Deux chapitres sont consacrés au servage. Apprécié par Herzen, qui publia en traduction la correspondance de Dolgoroukov avec le gouvernement russe, le livre fut considéré comme un «acte d'accusation contre sa patrie ${ }^{15} »$. N. Ejdel'man écrit :

Jamais encore ne s'était trouvé dans une opposition aussi résolue et dans l'émigration un homme à la fois d'aussi haute naissance et aussi bien renseigné. [...] On imagine maintenant difficilement que dans les années 1860, pour beaucoup de ses amis et ennemis, le nom de Dolgoroukov était placé à côté de celui de Herzen, quasiment à égalité avec lui. Plus encore, les autorités suprêmes craignaient même plus Dolgoroukov que Iskander ${ }^{16}$.

11 Déchu de ses titres, privé de ses biens, condamné à l'« exil perpétuel » (večnoe izgnanie) par un oukaze du Sénat du 5 juillet 1861 publié en 1862, interdit de séjour en France, Dolgoroukov passe en Belgique en juin 1862, où il publie plusieurs feuilles d'opposition constitutionnelles-monarchistes, dont Le véridique, sur la première page duquel on peut lire: "Cette revue a l'honneur d'être prohibée en France.» Il se réfugie ensuite en Angleterre, où il collabore à La cloche de Herzen, puis en Suisse en 1864. Les Russes le 
fuient comme la peste, «qui par peur du gouvernement russe, qui par dégoût $t^{17}$ ». Entretemps, en 1863, avait été lancée, contre Dolgoroukov et Ivan Gagarine, devenu jésuite et lui aussi déchu de tous ses titres, l'affaire des libelles anonymes qui provoquèrent le duel fatal de Pouchkine ${ }^{18}$. Les analyses graphologiques sont contradictoires, mais le contexte de l'apparition de ces accusations, 27 ans après les faits, les rend douteuses. Certains de ses contemporains ne pardonnaient pas à Dolgoroukov d'avoir révélé les secrets de famille de la noblesse, les liaisons et les enfants illégitimes, les faux titres de noblesse, les vices de Pierre le Grand, l'assassinat de Paul I ${ }^{\text {er }}$, etc. ${ }^{19}$. Bref, on a déjà là une véritable guerre de kompromaty, qui ne doit pas oblitérer l'intérêt des analyses et des propositions de Dolgoroukov.

On trouve aussi Nicolas Ivanovitch Sazonov (1815-1862), « doyen des émigrés russes ${ }^{20}$ », lui aussi privé en 1850 de tous ses titres et biens, mais rétabli dans ses droits en 1858, socialiste utopique, rédacteur en chef à Paris de La Gazette du Nord, « revue hebdomadaire internationale » fondée en octobre 1859 par Gabriel de Rumine ${ }^{21}$ et Sazonov (Sasonoff), qui cessa de paraitre fin juin 1860. Sazonov est critique envers Dolgoroukov, tout en étant proche de Herzen, auquel il consacra un grand $\operatorname{article}^{22}$. Sazonov se défend toutefois d'être un révolutionnaire :

J'aime assurément mieux la liberté que l'esclavage, le droit que l'arbitraire, l'ordre légal que le caprice personnel, le progrès que la stagnation, la science que les préjugés ; et pour tout dire en un mot, j'aime mieux Alexandre II qu'Ivan IV. Et voilà comment je suis révolutionnaire ${ }^{23}$.

Dans une série d'aricles sur l'émancipation des serfs publiée dans La Gazette du Nord, Sazonov donne un historique du servage, puis expose les différents projets en présence et l'état de la quetion tel qu'il ressort des procès-verbaux de la commission de rédaction, en discutant certains points. Il approuve le désir de la commission de ne pas transformer les 22 millions de paysans serfs en prolétaires, et de ne pas léser non plus les propriétaires. Le 2 juin 1860 ( La Gazette du Nord, nº 22), il publia le projet d'émancipation.

Parmi les observateurs étrangers, on trouve un ancien consul des États-Unis, Fleishman, qui propose à la Russie de s'inspirer de l'expérience de l'Amérique du Nord, la Russie possédant «tous les éléments d'une prospérité analogue » (2, p. 14); Auguste Jourdier, qui a passé "deux années d'études sur place », a été bien documenté par Milioutine, et qui s'appuie sur les «vingt et quelques » volumes des comités de rédaction dont il s'est fait traduire « les parties les plus saillantes » $(32, \mathrm{p} .31)$ pour faire une analyse critique des dispositions du projet final ; il préconise une réforme progressive, diversifiée selon les régions et les catégories de paysans, et critique les "superstitions slavophiles" de Samarine (p. 27) et l'esprit qui a présidé aux réformes : «l'esprit militaire qui veut l'unité en tout», «l'esprit démocratique mal entendu», et «l'esprit bureaucratique bien intentionné [...], mais peu éclairé » (32, p. 37).

Il y a aussi Alexandre Dumas, qui écrit en introduction à ses Lettres sur le servage en Russie, en septembre 1858 :

Au bout de trois mois, après avoir causé avec les hommes qui ont poussé l'empereur à rendre la loi d'émancipation, et avec les esclaves en faveur desquels elle a été rendue, avec les journalistes qui l'ont provoquée, et avec les propriétaires qu'elle frappe, je crois pouvoir donner des renseignements exacts sur son effet présent et ses conséquences à venir. $(9$, p. 120)

Mais seules les douze pages finales traitent de cette question, tout ce qui précède, soit 138 pages étant consacré à un historique du servage à Rome, en France au moyen âge et en Russie. 
Il n'est pas possible d'analyser ici toutes ces brochures. On en dégagera seulement les principaux thèmes et les principales propositions : les raisons de l'abolition du servage, la question de la terre à concéder aux paysans, celle du rachat et de ses modalités financières, la place de la commune paysanne, et enfin les conséquences prévisibles de l'abolition du servage.

\section{Pourquoi abolir le servage?}

17 Il y a d'abord des raisons morales et économiques : Nicolas Tourguenev parle du "péché » inhérent à la possession de son semblable. Le prince Henry de Valori est seul à se proposer de « démontrer, la Génése et les saintes Écritures à la main, que non seulement le servage, mais même l'esclavage n'est opposé ni aux lois divines ni aux notions de justice humaine » $(26$, p. 10) : la Russie n'est pas encore mûre pour l'affranchissement complet et sans transition de " 22 millions d'hommes qui ne sont ni moralisés, ni civilisés » $(26$, p. 7$)$.

Viennent ensuite les raisons économiques: le travail libre est beaucoup plus productif que la corvée. Pour Wolowski, membre de l'Institut, auteur dans La revue des deux mondes d'un revue de 17 publications sur l'émancipation des serfs, il s'agit de réaliser les idées de philanthropie et d'humanité, mais aussi de " conquérir l'instrument le plus énergique de la prospérité des nations modernes, l'activité volontaire » (7, p. 318). La servitude a dégradé les mœurs, « l'esclavage avilit à la fois l'esclave et le maître » (p. 319). C'est aussi la pensée de Dolgoroukov, qui dénonce avec le plus de véhémence le lien entre le servage et le despotisme. Il estime que la noblesse, ayant asservi ses concitoyens, a été elle-même asservie : elle est soumise à l'abitraire du pouvoir et de la police secrète, et à la rapine des fonctionnaires. Comme l'écrivait Dolgoroukov dans La vérité sur la Russie, "les droits civiques du dernier d'enre les portefaix d'un pays constitutionnel sont incontestablement, et de beaucoup, au-dessus des privilèges d'un noble russe » $(18$, p. 6). La noblesse doit renoncer à ses privilèges illusoires pour obtenir liberté et droits politiques.

Certains sont cependant réservés ou prudents. Ainsi, Schédo-Ferroti pense qu'«il est certain que la grande majorité des serfs en Russie est très contente de son sort » $(1$, p. 55). "Cependant, il est temps de s'occuper de la libération des serfs en Russie ; mais il serait absurde de prétendre qu'on puisse le faire de but en blanc et au moyen d'une simple signature, et d'un "byt' po semu" » (ainsi soit fait !).

\section{La question de la terre}

La première question discutée est celle de la terre : faut-il libérer les paysans avec la terre, et avec quelle terre?

Dolgoroukov défend le principe d'une émancipation avec une « dotation territoriale »:

Quoique, en principe, la terre appartienne au seigneur, néanmoins, en Russie, il aurait été éminemment dangereux d'émanciper les paysans sans leur accorder une dotation territoriale. Leur refuser cette dotation, ce serait créer en Russie vingtdeux millions de prolétaires, condamner au prolétariat le tiers de la population de l'empire, et organiser en Russie une Irlande sur une plus vaste échelle. Ç'aurait été d'une haute imprudence, d'autant plus que le paysan russe n'entend point se laisser émanciper sans terre. D'ailleurs, l'émancipation exonère le seigneur d'obligations 
souvent bien pesantes et bien onéreuses; il ne sera pas obligé de répondre pour les impôts de ses paysans, de les nourrir en cas de disette, de leur acheter des chevaux et du bétail en cas d'épizootie ; enfin, le travail libre sera beaucoup plus productif que ne l'était la corvée. Des expériences faites à cet égard ont prouvé que deux travailleurs libres, médiocrement actifs, font, dans l'espace d'une semaine, la

besogne de cinq serfs laborieux. (18, p. 8-9) 
vendre les domaines de la couronne et d'émettre des obligations pour pouvoir dédommager les propriétaires (18, p. 69). Comme il le raconte dans La vérité sur la Russie, c'est son cousin, Basile Dolgoroukov, chef de la police politique, qui demanda d'exclure ce point du projet pour pouvoir le publier ${ }^{25}$. Dolgoroukov reprocha à la commission d'êre constituée uniquement de partisans d'un rachat étalé dans le temps. Ce fut, selon Baxrušin, l'une des causes de l'émigration de Dolgoroukov, déçu dans ses ambitions de conseiller du prince, dépité de ne pas avoir obtenu de rôle digne de son rang nobiliaire, bloqué dans sa carrière par le système de la table des rangs, qu'il dénonçait vigoureusement dans La vérité sur la Russie.

Sazonov, au contraire, critique le projet prévoyant d'indemniser directement les petits propriétaires (21 serfs ou moins) et en même temps de venir en aide aux paysans

qui voudront acheter aux seigneurs les terres dont ils ont la jouissance, au moyen des sommes prélevées sur les revenus des domaines de l'État, et qui se montent à un total de 5000000 de roubles argent (20000 000 de francs). Tout cela n'a pas le caractère juridique, légal. En persévérant dans cette voie, on arrive, en théorie, à une confusion complète de tous les principes, et, en pratique, au maintien réel du servage, quoique sous une forme atténuée (La Gazette du Nord, $\mathrm{n}^{\circ} 7,18$ février 1860).

Le projet de Nicolas Tourguenev, qui souhaite des mesures promptes et décisives, est plus radical : pas de rachat, ni par le gouvernement, ni par les paysans; concession de 3 ha maximum par famille ou 1 ha $1 / 5$ par âme $(17$, p. 41), enclos y compris (c'est ce qu'il appelle la " petite concession ", qui coûterait 300 millions de roubles à l'État), opposée à la "grande concession", qui entraine des rapports conflictuels entre paysans et prorpriétaires. «Si l'on donnait aux paysans eux-mêmes le choix entre le mode de petite concession, tel que je le propose, et le mode de grande concession avec rachat, je suis convaincu qu'ils aimeraient mieux obtenir peu gratis, que beaucoup en payant ». Il admet l'apparition d'une classe d'ouvriers agricoles, de prolétaires (25, p. 69), mais rien n'empêchera ensuite les paysans, quand ils en auront les moyens, d'acheter des terres (25,p. 70).

31 Le prince Sanguszko ${ }^{26}$ s'inquiète du sort de la noblesse, qui doit conserver la position qu'elle occupe depuis des siècles $(21$, p. 3). Il propose l'abolition des corvées, la liberté d'installation des paysans, qui reçoivent en cadeau leur cabane et leur enclos, les terres restant revenant au seigneur. Il conseille de commencer par expérimenter la réforme dans les provinces de Kiev, Volhynie, Podolie, et éventuellement Grodno, proches de la Galicie, où l'émancipation a été pratiquée avec succès en 1848.

L'auteur de la Lettre d'un panslaviste à S. M. Alexandre II, qu'un indice, une référence à un livre de lui, permet d'identifier comme étant le comte Venceslas (Waclaw) Jablonowski, craint que la réforme ne soit une "tentative calculée de décomposition sociale » (13, p.13), qu'elle n'entraîne la ruine de la petite noblesse. Il préconise un système de métayage, sur un tiers des terres, un autre tiers étant acheté par les communes, et le dernier tiers étant exploité à sa guise par le propriétaire. Un tiers-état agricole devrait naître de ce système. On notera chez lui une appréciation favorable du rôle que pourraient jouer les fermiers juifs.

Alexandre Dumas distingue trois partis, et deux nuances qui portent sur le rôle du mir, et il expose les vues des différents camps : les « réactionnaires contre-émancipateurs; parti peu considérable, mais bien appuyé à Saint-Pétersbourg », le parti du juste milieu, celui des propriétaires qui « admettent l'émancipation, mais l'émancipation progressive, et qui ne veulent pas donner l'enclos, convaincus qu'ils ne seront jamais payés par les paysans ", 
et enfin « les progressistes, les journalistes, les gens de lettres, les employés, la bohême intelligente enfin, qui veulent l'émancipation à tout prix, comme un retour vers le sens moral, comme une expiation de trois siècles d'injustice et d'oppression » (9, p. 261). Dumas ne prend pas position, mais estime que « donner la liberté tout de suite et l'enclos pour rien » est peut-être «le moyen le plus simple et même le moins dangereux » (9, p. 271).

\section{La question financière} 27). « décompter 425 millions de roubles qui représentent la somme des hypothèques de l'État sur les biens de la noblesse ${ }^{27} »(18$, p. 9). Il propose un emprunt de 200 millions de roubles, et ce qu'on appellerait maintenant des privatisations :

L'on aurait pu vendre pour 375 millions de roubles de domaines de la couronne, usines, fabriques, salines, pêcheries, prairies, forêts (dont l'État possède plus de cent millions d'hectares). Le chemin de fer de Saint-Pétersbourg à Moscou seul aurait pu être vendu pour 70 à 80 millions de roubles. En attendant que toutes ces ventes s'effectuassent, l'on aurait pu émettre, pour cette somme, des obligations portant intérêt [...] Le véritable obstacle à tout cela, c'est que les domaines de la couronne sont administrés par la bureaucratie, qui les exploite, les pille et ne veut point les laisser échapper. Or, la bureauratie et sa protectrice la camarilla sont les souverains de la Russie, dont l'empereur n'est que l'autocrate nominal ${ }^{28}$.

Schédo-Ferroti rejette d'avance ce que proposera Dolgoroukov et estime le coût pour l'État à 3 milliards de roubles d'argent. Il prévoit de lancer un emprunt en France pour 250 millions (1, p. 16).

\section{La question du mir}


C'est la commune russe, où "l'homme du peuple respire à l'aise sous l'égide d'une administration dont il a choisi lui-même le personnel, et qui lui inspire de la confiance par la simplicité même de son mécanisme, dont il voit et comprend le jeu » (1, p. 59). Il faut faire passer graduellement les paysans des terres seigneuriales sous le système de la commune. Les paysans conserveraient leurs obligations de corvée envers les seigneurs terriens, mais ceux-ci cèderaient leurs droits de basse justice à la commune (1, p. 60). Schédo-Ferroti propose, en 1857, de proclamer un premier oukase, abolissant la petite propriété, et par là le servage personnel; puis viendrait la fondation de banques provinciales et la création de biens nobles. En 1860, les biens seigneuriaux seraient groupés en communes de 1500 à 2000 âmes, 3 à 4 de ces communes formant un canton ( volost'). Un premier pas sur la voie de l'affranchissement serait d'autoriser le paysan à changer de village à l'intérieur de sa commune (en 1865), en renonçant à son lot de terre et à sa maison, «lesquels doivent rester en la possession du seigneur, dont ils sont la propriété incontestable» (1, p. 61). Puis viendrait le droit de translation d'une commune à l'autre dans les limites du canton (1871), puis dans celles de la province (en 1874) et enfin de l'Empire (en 1877).

41 Comme les populistes, Sazonov imagine un système associatif de communes (comprenant les habitants de toutes les classes):

Si les terres communales étaient définitivement partagées en lots individuels, au bout de très peu de temps, les capitalistes parmi les paysans (il y en aussi en Russie) absorberaient les parcelles appartenant aux cultivateurs les plus pauvres, ceux-ci devenant des prolétaires [...] Tous devenus des cultivateurs libres et propriétaires, [ils] formeront des agglomérations de forces productives et de capitaux, où le principe de l'association se trouvera appelé immédiatement à jouer un rôle prépondérant $^{30}$.

Pour Nicolas Tourgueneff, il doit y avoir une seule administration pour les paysans, sur la base du canton (15000 âmes), le self-governement des communes étant conservé (25, p. 50 et suiv.). Tous les paysans ci-devant seigneuriaux seront soumis à l'administration communale exclusivement $(25$, p. 57$)$. Les propriétaires indépendants appartiendront au canton.

Haxthausen, l'« inventeur » du mir, en fait un panégyrique : c'est l'

organisation communale des villages russes [qui] garantit mieux que toute autre à tous les membres de la commune l'ordre et la sécurité de la propriété; elle raffermit la vie de famille [...], elle maintient dans leur vigueur les vertus politiques et sociales, elle fortifie les sentiments d'homogénéité, de communauté, de fraternité, de justice, de l'amour du pays et du clocher (3, p. 11).

Pour Wolowski, de l'Institut, au contraire, ce communisme rural est le «produit d'une organisation oppressive» (7, p. 326). «L'homme, non responsable, se reposant sur la commune, reste endormi dans l'imprévoyance [...] Avec la loi agraire du communisme, l'homme est absorbé par la masse, son individualité sommeille» (7, p. 619). Il critique Gérebtzoff ( $C$ C'est au contraire parce que la Russie possède un fonds de commmunisme qu'elle reste en arrière du monde civilisé », 7, p. 626).

Il s'agit non seulement de briser les liens du servage, mais encore d'asseoir le droit permanent de la propriété du sol. Il faut rompre à la fois avec l'esclavage et avec le communisme, ces deux instruments de la dégradation des âmes. La grande réforme dont l'empereur Alexandre II a pris la généreuse initiative ne sera sérieuse et complète qu'à cette condition. $(7, \mathrm{p} .631)$ 

classe supérieure, c'est faire preuve d'une grande naïveté ! [...] En Russie, il n'existe point d'institutions politiques, l'arbitraire et le caprice en tiennent lieu. Le gouvernement devrait créer ces institutions; il devrait, dans le plein exercice de son pouvoir monarchique, accorder une constitution à ses sujets, qui veulent cesser d'être esclaves et tiennent à devenir des hommes libres. Si le gouvernement n'accorde point une constitution de plein gré, d'ici à quelques années le courant des choses en amènera une, mais peut-être au prix d'événements déplorables que tout bon Russe voudrait éviter. L'empereur Alexandre, prince animé des meilleures intentions, consentira-t-il à octroyer une constitution? En présence de son déplorable entourage, l'on n'ose point l'espérer ${ }^{32}$. 
permis d'attribuer certaines brochures anonymes), on notera le caractère sans précédent de ce débat international, auquel prirent part non seulement des Russes, mais aussi des étrangers ${ }^{33}$, cette participation d'experts étrangers étant acceptée comme quelque chose de normal. Il y eut un véritable pluralisme d'opinions, certaines propositions étant tout à fait modernes, comme les privatisations envisagées par P. Dolgoroukov. Le lecteur étranger ne pouvait rien ignorer de l'histoire du servage, de ses formes, des problèmes liés à son abolition, avec parfois des exposés très techniques pour les aspects économiques et financiers de la question. Tous les auteurs ont le souci du bien commun et la prospérité de la Russie. La variété des propositions, le haut niveau de la discussion rendent ce débat exemplaire. L'influence de ces brochures et ouvrages sur le comité de rédaction fut sans doute minime, le gouvernement sachant cependant qu'il se trouvait sous la surveillance de l'opinion publique internationale. Et ces débats d'il y a 150 ans sur l'état de la Russie et son avenir nous paraissent souvent bien actuels.

\section{BIBLIOGRAPHIE}

1. Études sur l'avenir de la Russie. Première étude : La libération des paysans, par D. K. Schédo-Ferroti [pseudonyme de F. I. Fircks], Berlin, B. Behr, 1858, 80 p. (daté de Moscou, mars 1857).

ILCEA, 17| 2013 
2. Les États-Unis et la Russie considérés du point de vue de la grande culture et du travail libre, par Ch. L. Fleishmann, ancien consul des États-Unis, Librairie A. Franck, 1858, 64 p.

3. De l'abolition par voie législative du partage égal et temporaire des terres dans les communes russes par le baron Auguste de Haxthausen, Librairie A. Franck, 1858, 19 p.

4. Les Questions du jour en Russie : abolition du servage, liberté individuelle, publicité, par Olguerdovitch [pseudonyme du prince Nikolaï Ivanovitch Troubetzkoy, 1807-1874], E. Dentu, 1858, II-80 p. (daté de février 1857).

5. De l'abolition du servage en Russie, examen critique du projet de l'Empereur Alexandre II. Conséquences politiques et sociales des dispositions décrétées dans les rescrits impériaux et dans les offices ministériels du 21 novembre-3 décembre et du 5-17 décembre 1857, par Louis Lubliner, avocat à la cour d'appel de Bruxelles, Bruxelles, Imprimerie de Ch. Vanderauwera, 1858, 91 p. [Ludwik Ozeasz Lubliner].

6. De la production agricole, par M. D. Stolypine, Paris, Amyot, 1858, 32 p.

7. L. Wolowski, de l'Institut, « La question du servage en Russie. I - Nécessité de l'émancipation et condition actuelle des serfs », Revue des deux mondes du 15 juillet 1858, p. 317-349; « II - Les paysans de la couronne. Le communisme russe ", Revue des deux mondes, $1^{\text {er }}$ août 1858, p. 595-631. (Étude basée sur dix-sept publications en français, allemand, russe et polonais, de 1847 à 1858, parmi lesquelles : Du développement des idées révolutionnaires en Russie, par Iscander (Hertzen), Paris, 1854 ; Les trois questions du moment, par Nicolas de Gerebtzof, Paris, 1857 ; Kolokol, Londres, 1858 ; Stolypine [6], Lubliner [5], Olguerdovitch [4], Schédo-Ferroti [1].)

8. Réflexions préalables sur les bases proposées au mode d'émancipation des serfs en Russie, par un député d'un Comité provincial, P. Guillaumin et C ${ }^{\mathrm{ie}}, 1859,47$ p. [comte Vladimir Petrovitch Orlov-Davydov (1809-1878), membre d'honneur de l'Académie impériale des sciences].

9. Dumas Alexandre, Lettres de Saint-Pétersbourg [sur le servage en Russie]. Bruxelles, Rozez, 1859, 232 p. Édition autorisée pour la Belgique et l'étranger, interdite pour la France [édition consultée : Lettres sur le servage en Russie, in A. Dumas, Impression de voyage en Russie, t. IV, Paris, Calmann Lévy, 1884, p. 120-271]. Daté de Saint-Pétersbourg, 19 septembre (1 ${ }^{\text {er }}$ octobre 1858).

10. Émancipation des serfs en Russie. Examen du projet financier de MM. Fraenkel et Homberg, banquiers, suivi du résumé d'un autre projet relatif au même projet, Bruxelles et Leipzig, C. Muquardt, 1859, 32 p.

11. De l'émancipation des serfs en Russie (état actuel de la question), par Nicolas de Gerebtzoff, Paris, Amyot, 1859, 47 p. [Paris, janvier 1859].

12. Lettres russes. Alexandre II et l'émancipation, par A. Grandguillot, Paris, E. Dentu, 1859, 160 p.

13. Lettre d'un panslaviste à S. M. Alexandre II, empereur de toutes les Russies, roi de Pologne à propos de l'affranchissement des serfs, déposée entre les mains des membres de la Commission supérieure nommée pour régler les bases de l'émancipation des serfs, Paris, E. Dentu, 1859, 36 p. [comte Venceslas Jablonowski].

14. Lettre d'un député de comité à Monsieur le président de la commission de rédaction, aide de camp général Rostovtzoff, Paris, Guillaumin et $\mathrm{C}^{\mathrm{ie}}, 1859,47$ p. [comte Orlov-Davydov, cf. $\mathrm{n}^{\circ} 8$ ].

15. L'Émancipation de la Russie, considérée au point de vue de l'économie politique, par Jules Pautet, Paris, Guillaumin, 1859, 15 p. (extrait du Journal des économistes, $n^{\circ} 66$, juin 1859).

16. Études sur la question de l'abolition du servage en Russie, par un contemporain [Th. de Thoerner], Paris, Librairie internationale de l'office du Nord, 1859, 360 p.

17. N. Tourgueneff, Encore un mot sur l'émancipation des serfs en Russie, Paris, Amyot, 1859, 20 p. 
18. De la question du servage en Russie, par M. le Prince Pierre Dolgoroukow (extrait du Journal des économistes, décembre 1860), Paris, Guillaumin et $\mathrm{C}^{\mathrm{ie}}, 1860,11 \mathrm{p}$.

19. La vérité sur la Russie, par le prince Pierre Dolgoroukow, Paris, A. Franck, 1860, $306+310$ p.

20. Régénération sociale de la Russie, par Victor de Porochine, ancien professeur, membre du Comité d'émancipation du gouvernement de Grodno, Paris, A. Bourdillat, 1860, VIII-299 p.

21. De l'émancipation des paysans en Russie, par le prince Ladislas Sanguszko, manuscrit imprimé, tiré à soixante exemplaires, 22 p. [Vienne, mars 1860].

22. Sasonoff, « De l'émancipation des serfs en Russie », La Gazette du Nord, $\mathrm{n}^{\circ} 10$ (10 décembre) et $\mathrm{n}^{\circ} 13$ (31 décembre) 1859, $\mathrm{n}^{\text {os }} 1,3,4,7,9$ des 7, 21, 28 janvier, 18 février et 3 mars 1860.

23. De l'émancipation des serfs en Russie par un publiciste russe (extrait du journal Le Nord), Paris, Guillaume et $\mathrm{C}^{\mathrm{ie}}, 1860,59 \mathrm{p}$.

24. Lettres sur l'émancipation des serfs (insérées dans « le Nord»), par D. S. [Dimitri Stolypine ?, cf. nº 34]. Paris, Office du « Nord », 1860 [non consultable à la BNF].

25. Un dernier mot sur l'émancipation des serfs en Russie, par N. Tourgueneff, Paris, A. Franck, 1860, $114 \mathrm{p}$.

26. La question russe, en réponse au parti ultra-libéral de la Russie, par le prince Henry de Valori, Paris, E. Dentu, 1860, 23 p.

27. Études financières sur l'émancipation des paysans en Russie, sur l'impôt foncier, le système monétaire et le change extérieur, par Alexandre Zapasnik, Paris, A. Franck, 1860, 172 p.

28. Quelques remarques sur la question de l'affranchissement des paysans en Russie, Leipzig, Brockhaus, $1861,32 \mathrm{p}$.

29. Le manifeste impérial d'émancipation ; lettre à S.M.I. de toutes les Russies par un paysan obligé, Paris, E. Dentu, 1861.

30. L'Église russe a-t-elle fait quelque chose pour l'affranchissement des paysans en Russie ?, par un Russe, Genève (20 octobre 1861), H. Georg, 1861, 38 p.

31. L'émancipation des serfs en Russie, par le prince Augustin Galitzin,extrait du Correspondant, Paris, Charles Douniol, 1861, 22 p. (Revue de plusieurs études : Régénération sociale de la Russie, par V. Porochine $\left[=\mathrm{n}^{\circ} 20\right]$; Études sur la question de l'abolition du servage en Russie, par un contemporain [= $\left.\mathrm{n}^{\circ} 16\right]$; Matériaux pour servir à l'histoire de l'abolition des serfs en Russie, Leipzig, 1860 (en russe) ; Dolgoroukow [18], Zapasnik [27], Jourdier [32].)

32. De l'émancipation des serfs en Russie, état de la question au 16 mars 1861 : exposé et critique des projets dits du comité de rédaction. Avec une carte et des tableaux statistiques, par Auguste Jourdier, auteur des Forces productives, destructives et improductives de la Russie et d'un Voyage agronomique en Russie, de 1859 à 1861, etc., A. Franck, 1861, 80 p.

33. Solution pratique de la question des paysans en Russie, par Victor de Porochine, ancien professeur d'économie à l'Université de Saint-Pétersbourg, Paris, Bourdillat, 1861, 24 p.

34. De l'industrie agricole. Émancipation des serfs en Russie, par D. S. Leipzig, A. Franck'sche VerlagsBuchhandlung, 1861, 70 p. [Dmitri Stolypine].

35. Études sur l'avenir de la Russie. Sixième étude : Les serfs non encore libérés, par D. K. SchédoFerroti, Berlin, B. Behr, 1861, 100 p. [sur les serfs des mines d'or de Nertchinsk].

36. L'abolition du servage en Russie, par M. Boris Tchitcherine, Imprimerie de H. Plon, 1861, 23 p. (extrait de La critique française, 1861, t. 1). 
37. Des réformes en Russie, suivi d'un aperçu sur les États généraux russes au XVI et au XVII siècles, par le prince Pierre Dolgoroukow, Paris-Bruxelles, 1862, 332 p.

\section{NOTES}

1. Selon Sazonov, le terme d' "émancipation" a été officiellement utilisé pour la première fois par le général Kokovcov, président du comité de rédaction, dans sa réponse à une lettre du prince Orlov du 17 février 1859, au lieu de l'expression " amélioration du sort des paysans » jusqu'alors en usage (La Gazette du Nord, $\mathrm{n}^{\circ}$ 3, 21 janvier 1860 ; voir bibliographie, $\mathrm{n}^{\circ} 22$ ).

2. Le premier chiffre renvoie à la bibliographie finale.

3. Disponibles sur <http://books.google.fr/>.

4. Sbornik postanovlenij i rasporjaženij po cenzure s 1720 po 1862, SPb., Tip. Morskogo Ministerstva, 1862 , p. 422 (circulaire du 16 janvier 1858); A. M. Skabičevskij, Očerki istorii russkoj cenzury (1700-1863), SPb., 1892, p. 420.

5. Sbornik postanovlenij, p. 426.

6. Ibid., p. 441.

7. A. M. Skabičevskij, p. 422.

8. Ibid., p. 425.

9. Le nouveau code (provisoire) de la censure (du 12 mai 1862) limitera la publication de textes sur les manquements et les malversations de l'administration aux ouvrages d'au moins dix feuilles d'imprimerie et aux périodiques dont l'abonnement n'était pas inférieur à sept roubles par an (Sbornik postanovlenij, p. 469), ce qui excluait les éditions à bon marché pour le peuple, qui devait être "protégé ». La critique "en général » des statuts du 18 février sera aussi interdite (p. 472).

10. Sbornik postanovlenij, p. 444.

11. Lettre de Herzen à P. Dolgorukov du 12/31 mars 1860, Sobranie sočinenij v 30 tomax, t. XXVII, 1 , p. 36.

12. On trouvera une dizaine de références d'articles de journaux (1858-1859) dans l'article de Mihail D. Sturdza, «Bibliographie d'un réquisitoire. La Russie vue par la presse parisienne, juillet 1848-juillet $1859 »$ ", Cahiers du monde russe et soviétique, 1968, n 9 (3-4), p. 423-424.

13. Voir le portrait de P. Dolgoroukov (surnommé « le Bancal») dans la lettre de G. Samarine à I. Gagarine du 19 juillet 1840 (Ivan Gagarine - Georges Samarine. Correspondance 1838-1842, éd.

F. Rouleau et S. Galievsky, Meudon, Plamia, 2002, p. 116-117).

14. Mix. Lemke, Nikolaevskie žandarmy i literatura, 1826-1855 gg, SPb., 1909, p. 527-552.

15. Kolokol, $\mathrm{n}^{\circ}$ 73-74, 15 juin 1860.

16. N. Ejdelm'an, Gercen protiv samoderžavija, M. Mysl', 1973, p. 307, 312.

17. S. Baxrušin, " "Respublikanec-knjaz"” Petr Vladimirovic Dolgorukov », in P. V. Dolgorukov, Peterburgskie očerki. Pamflety èmigranta 1860-1867, Moscou, Novosti, 1992, p. 35 (rééd. de l'édition de 1934). Cf. I. N. Ermolaev, Žizn' i bor'ba knjazja Petra Dolgorukova, Pskov, Puti russkogo liberalizma, 2001, $440 \mathrm{p}$.

18. Pour l'histoire de ces bruits, et de leur réfutation, l'article le plus circonstancié est celui de A. S. Buturlin, «Imel li I. S. Gagarin otnošenie k paskvilju na A. S. Puškina? », Izvestija OLJa 28, 3, 1969, p. 277-285. La correspondance Gagarin-Dolgorukov a été publiée dans Simvol, 13, 1985, p. 210-253 par L. Shur.

19. S. Baxrušin, art. cité, p. 41-42.

20. M. Cadot, La Russie dans la vie intellectuelle française, 1839-1856, Fayard, 1967, p. 32 et p. 31-34 pour un portrait de Sazonov. 
21. 1841-1871. Il légua une partie de sa fortune à la ville de Lausanne, qui fit construire le «palais de Rumine ", abritant musées et bibliothèque.

22. La Gazette du Nord, $\mathrm{n}^{\circ}$ 21, 26 mai 1860. Des lettres de Sazonov à Herzen ont été publiées dans Literaturnoe nasledstvo, t. 62, M. 1955, p. 522-545.

23. La Gazette du Nord, $\mathrm{n}^{\circ} 12,24$ décembre 1859, p. 3. Sazonov est l'auteur (anonyme) de La vérité sur l'empereur Nicolas : histoire intime de sa vie et de son règne, par un Russe, Paris, Librairie nouvelle, 1854,319 p.

24. Baxrušin, art. cité,p. 19. Tourgueneff, Un dernier mot..., 25, p. 5.

25. P. Dolgoroukov, La vérité sur la Russie, Franck, 1860, t. 2, p. 48 ; Baxrušin, art. cité, p. 20.

26. Prince Ladislas Sanguszko-Lubartowicz (Slawuta, 1803 - Cannes, 1870).

27. Les mêmes chiffres sont cités par Sazonov (La Gazette du Nord, $\mathrm{n}^{\circ} 11,17$ décembre 1859), d'après le procès-verbalde la commission de rédaction du 20 mai 1859.

28. 18 , p. 10. Le "député d'un comité provincial » estime aussi que la bureaucratie a intérêt à compliquer la question de l'émancipation ( $n^{\circ} 8$, p. 41).

29. Les trois questions du moment, par Nicolas de Gérebtzoff, E. Dentu, 1857, p. 66. Outre cette question du « communisme », les deux autres questions sont celle du libre-échange (qui doit être régulé par un "pouvoir supérieur»), et celle des chemins de fer. Il n'est pas encore question du servage dans cette brochure, qui espère pour la Russie un « merveilleux avenir ».

30. La Gazette du Nord, $\mathrm{n}^{\circ}$ 9, 3 mars 1860.

31. Questions agricoles concernant la terre et le capital, par M. D. Stolypine, Paris, Amyot, 1857, p. 24.

32. 18, p. 10-11; cf. P. Dolgorukov, O peremene obraza pravlenija v Rossii, Leipzig, Franck, 1862.

33. Il circula même en Russie une fausse lettre de Guizot, mettant en garde contre les conséquences du servage (N. Barsukov, Žizn' i trudy M. P. Pogodina, kn. 16, SPb., 1902, p. 74-75).

\section{RÉSUMÉS}

Présentation et analyse d'une quarantaine de brochures en français de 1858-1861 sur l'émancipation des serfs en Russie, publiées par des Russes ou des étrangers de toutes tendances. Les principales questions abordées sont: les raisons de l'abolition du servage, la question des terres, de leur rachat éventuel, la place du mir, les conséquences de la réforme. Cette discussion internationale d'un projet gouvernemental russe est sans équivalent dans l'histoire russe.

Presentation and analysis of about forty brochures in French of 1858-1861 on the emancipation of the serfs in Russia, published by Russians or foreigners of different ideologilcal trends. The main approached questions are: the reasons of the abolition of the serfdom, the question of allotment, the possible repurchase of him, the place of the mir, the consequences of the reform. This international discussion of a Russian governmental project is unprecedented in Russian history.

ОБЗОР ОКОЛО 40 БРОШЮР 1858-1861 ГГ. НА ФРАНЦУЗСКОМ ЯЗЫКЕ ОБ ЭМАНСИПАЦИИ КРЕПОСТНЫХ КРЕСТЬЯН В РОССИИ, ОПУБЛИКОВАННЫХ НА ЗАПАДЕ РУССКИМИ ИЛИ ИНОСТРАННЫМИ АВТОРАМИ ВСЕХ ТЕНДЕНЦИЙ. ГЛАВНЫМИ ОБСУЖДАЕМЫМИ В НИХ ВОПРОСЫ ЯВЛЯЮТСЯ ПРИЧИНЫ ОТМЕНЫ КРЕПОСТНОГО ПРАВА, ВОПРОС О ЗЕМЛЕ И О ЕЕ ВОЗМОЖНОМ ВЫКУПЕ, ФУНКЦИИ МИРА, ПОСЛЕДСТВИЯ РЕФОРМЫ. ТАКОЕ МЕЖДУНАРОДНОЕ ОБСУЖДЕНИЕ ГОСУДАРСТВЕННОГО ПРОЕКТА НЕ ИМЕЕТ ЭКВИВАЛЕНТА В РУССКОЙ ИСТОРИИ. 
INDEX

Mots-clés : Russie, Alexandre Ier, abolition servage, émigrés, serfs, émancipation, commune, noblesse, ouvrages

Keywords : serfs, emancipation, peasant corporation, nobility, works

motsclesru РОССИЯ, АЛЕКСАНДР 1ЫЙ, ОТМЕНА, КРЕПОСТНОЕ ПРАВО, ЭМИГРАНТЫ

\section{AUTEUR}

MICHEL NIQUEUX

Université de Caen Basse-Normandie 